\title{
Carnets
}

Revue électronique d'études françaises de l'APEF

Deuxième série - 19 | 2020

Petite fabrique d'interprètes

\section{Interjection (Racine)}

\section{Franc Schuerewegen}

\section{(2) OpenEdition}

Journals

Édition électronique

URL : http://journals.openedition.org/carnets/11831

DOI : 10.4000/carnets. 11831

ISSN : 1646-7698

Éditeur

APEF

\section{Référence électronique}

Franc Schuerewegen, «Interjection (Racine) 》, Carnets [En ligne], Deuxième série - 19| 2020, mis en ligne le 31 mai 2020, consulté le 23 décembre 2020. URL : http://journals.openedition.org/carnets/ 11831 ; DOI : https://doi.org/10.4000/carnets.11831

Ce document a été généré automatiquement le 23 décembre 2020.

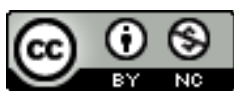

Carnets est mis à disposition selon les termes de la licence Creative Commons - Atribution - Pas d'utilisation commerciale 4.0 International. 


\title{
Interjection (Racine)
}

\author{
Franc Schuerewegen
}

Herminia je pense à toi.

Franc S.

1 1. L'épisode est touchant, et aussi un peu pénible; je rappelle de quoi il s'agit. Titus va se séparer de Bérénice, il essaie de le lui dire. Les mots, toutefois, ne viennent pas. Titus ne peut parler et quitte la scène, en invitant son confident à le suivre :

Sortons, Paulin, je ne lui puis rien dire. ${ }^{1}$

2 Ces paroles, quand on y réfléchit, sont curieuses. Titus ne peut rien dire à Bérénice. Or, à Paulin, il dit qu'il ne peut rien dire. Titus a donc bien dit quelque chose. En termes techniques : il y a contradiction entre l'énoncé et l'énonciation. Il est vrai que Bérénice n'est pas pour Titus, quand il prononce ces mots, une interlocutrice. Mais on a pu assister, juste avant ce moment, à un échange entre les amants et force est d'admettre que Titus, qui ne peut rien dire, n'est pas resté silencieux. L'amoureux « aphasique » - je rappelle que, pour Barthes, Bérénice est une «tragédie de l'aphasie ${ }^{2}-$ a parlé. Titus s'est exprimé. C'est ce que j'appelle, pour l'instant, la chose curieuse.

Certes, le problème auquel on a affaire est lié aux conditions de la scène. Au théâtre le silence est une autre sorte de parole. Un comédien qui se tait signifie son silence, qui est expressif. Racine en est conscient, d'une certaine manière, il triche. En somme, il n'a pas d'autre choix. Titus, donc, est aphasique et capable de verbaliser son aphasie. Il est les deux à la fois. Si Titus ne pouvait m'informer verbalement sur la nature de son problème, je ne saurais pas que ce problème existe. Bérénice est une tragédie, non une pantomime. Pour cela, Titus doit parler, donc, il parle.

4 Mais un autre élément nécessite notre attention, et qui va rendre l'enquête plus intéressante. Je reprends la même séquence pour une analyse plus détaillée. Titus aime Bérénice, celle-ci est inquiète. Titus l'évite, pourquoi le fait-il ? Serait-ce qu'il ne l'aime plus ? Bérénice lui pose la question, Titus ne peut répondre. A ce moment apparaît le blocage. La parole de Titus est une parole empêchée : 
Non, Madame. Jamais, puisqu'il faut vous parler,

Mon cœur de plus de feux ne se sentit brûler.

Mais...

5 L'empêchement a une cause, que l'on connaît. Dire « je t'aime », pour Titus, est aussi dire « je te quitte ». Les deux choses ne peuvent s'énoncer ensemble. Alors, mieux vaut rester silencieux. La suite est tout aussi connue, Barthes s'appuie sur elle pour établir le diagnostic d' « aphasie » :

Bérénice :

Achevez.

Titus :

Hélas!

Bérénice :

Parlez.

Titus :

Rome... L'Empire...

Bérénice :

Hé bien ? (II, 4)

Après quoi on trouve le "Je ne lui puis rien dire " que nous avons déjà cité et dont Bérénice se fait l'écho au début de la scène suivante :

Quoi me quitter si tôt, et ne me dire rien?

Chère Phénice, hélas! Quel funeste entretien! (II, 5)

Entretien rime avec rien. Racine cherche manifestement à enfoncer le clou. La scène 4 de l'acte II de Bérénice aura été, selon l'analyse que font les deux amants de leur situation, une scène pour rien. Mais est-ce vraiment le cas? Je reste dubitatif, je pense exactement le contraire. Il n'est pas vrai que Titus ne dise rien, Titus a parlé, il a dit des choses. Pour preuve, la séquence que nous venons d'examiner. Au vers 627, il prononce: « Rome... L'Empire... », au vers 625, il dit : « Hélas! ». Encore une fois : un comédien sur scène doit exprimer ses silences, il doit les jouer, car les silences font partie de son rôle. Or je découvre ici que le « Hélas! » de Titus est en somme bien plus efficace que son «Je ne lui puis rien dire ». Quand on dit qu'on ne peut rien dire, on dit quelque chose, on fait surgir un paradoxe. Quand on dit « Hélas! », on évite le paradoxe. En quelque sorte, dans les circonstances certes particulières auxquelles il est confronté, en disant « Hélas! », Titus s'exprime correctement.

8 2. Les «Hélas!", il convient également de le signaler, sont nombreux, et même très nombreux dans Bérénice. Quelques données chiffrées pour illustrer le propos. On en compte cinq, dans la seule scène que nous venons d'étudier ${ }^{3}$. Cinq « Hélas! " pour une seule scène, c'est beaucoup. Un coup d'œil sur la Concordance de Freeman et Batson m'apprend par ailleurs que les « Hélas! » sont au nombre de vingt-huit pour l'ensemble de la tragédie. C'est aussi le score maximal pour toutes les pièces de Racine. Comparons avec Andromaque : dix-huit «Hélas! », avec Britannicus : quinze, avec Bajazet : dix-sept, avec Phèdre: onze, avec Athalie : onze également, avec Esther: huit. Il est vrai que l'on trouve vingt-deux «Hélas!» dans La Thébaïde, chiffre également élevé mais on reste toujours en dessous des vingt-huit de Bérénice qui est donc championne en la matière ${ }^{4}$. Si on juge ce grand nombre alarmant, on pourra y ajouter un facteur aggravant. Racine a placé un ultime « Hélas! » dans la bouche d'Antiochus qui prononce, au vers 1518, le mot de la fin de la pièce. Ce « Hélas! » final a provoqué, comme on sait, l'ire de Voltaire qui le critique sévèrement dans ses «Remarques sur Bérénice». Voltaire écrit notamment : 
On peut être choqué qu'une pièce finisse par un hélas! Il fallait être sûr de s'être rendu maître du cœur des spectateurs pour oser finir ainsi. Voilà sans contredit la plus faible des tragédies de Racine qui sont restées au théâtre. ${ }^{5}$

Voltaire est "choqué ». Je suppose que s'il l'est, ce n'est pas seulement à cause du «Hélas! " placé en position finale mais aussi pour la série que le même « Hélas ! "vient clôturer. Pour Voltaire, c'est le « Hélas! » de trop, la goutte qui fait déborder le vase. Je note que Voltaire explique le «Hélas! » qui le choque, et donc, encore une fois, dans la lecture que je fais de son texte, qu'il juge nuisible parce que représentatif d'une trop longue série, par une intention d'auteur, intentio auctoris. Mais l'intentio est donc, pour Voltaire, négative. Plus exactement: selon Voltaire, Racine est conscient qu'en terminant sa pièce sur un «Hélas!", il fait un choix critiquable, en somme qu'il court le risque de passer pour maladroit. Mais il sait que son public lui est acquis, d'une certaine manière, qu'il peut tout se permettre. L'intention d'auteur de Racine, pour Voltaire, aura été, je le dirai un peu familièrement, de ne pas se mouiller la chemise.

3. L'objection que formule Voltaire, à mon humble avis, ne tient pas la route. Racine n'est pas du genre à se permettre des nonchalances, ni à commettre des maladresses. Il ne nous a pas habitués à cela. S'il est certes devenu, après Andromaque et Britannicus, un auteur respecté et reconnu, si son public l'aime, cela ne l'invite pour autant pas à se reposer sur ses lauriers. Bien au contraire, ajouterai-je. Ce qui l'intéresse, c'est la quête de la perfection. Toujours mieux faire, aller vers la quintessence du genre tragique. Devenir Racine, en somme. J'ai alors envie de reprendre l'argument de l'intentio auctoris, qui est aussi l'argument de Voltaire, mais en le renversant, c'est-à-dire en m'en servant ici de manière positive. L'auteur de Bérénice, dirons-nous, a fait exprès de multiplier les «Hélas!" dans sa tragédie, il a cherché à produire, en les multipliant, un effet esthétique. La multiplicité des «Hélas!» est voulue par Racine, elle n'est pas accidentelle, elle est stratégique.

11 Mon hypothèse sur l'intentio auctoris mérite un mot d'explication. Je suis ici obligé d'appeler Corneille à la barre. Comme toujours chez Racine, le " père de la tragédie » rôde dans les parages. Figure tutélaire, Corneille est aussi une présence un peu encombrante. Il semble que ce soit également le cas ici. On sait l'affaire des deux Bérénice. La pièce de Racine est créée sur la scène de l'Hôtel de Bourgogne le 21 novembre 1670. Une semaine plus tard, on joue, au Palais-Royal, une autre Bérénice dont l'auteur est Corneille, et qui sera publiée sous le nom de Tite et Bérénice. On a voulu croire qu'Henriette d'Angleterre avait fait travailler les deux hommes sur le même sujet, et à l'insu l'un de l'autre. Tout cela est loin d'être sûr. Je renvoie sur ce point aux explications de George Forestier en retenant seulement ici que, puisque Racine, au moment d'écrire sa pièce, a bien compris que Corneille est là et qu'il peut lui faire de l'ombre, il a intérêt à faire une Bérénice qui ne soit pas "cornélienne ", et qui se présente comme telle au regard du public ${ }^{6}$. Alors que fait-il?

12 Je propose le scénario suivant, j'essaie d'affiner mon hypothèse. Racine va chercher, chez Corneille, dans les textes de cet auteur, les matériaux qui vont lui permettre, justement, de ne pas être «cornélien » et, donc, de réussir une Bérénice pleinement « racinienne ». Comment procède-t-il ? Il avance en habile stratège qu'il est. Quand il écrit Bérénice, il ne connaît pas, pas encore le texte de Tite et Bérénice. En revanche, il se souvient du Cid, surtout, pour la question qui nous occupe, il a en mémoire une phrase de l'«Examen » du Cid qui va maintenant, alors qu'il commence Bérénice, lui être utile. Je rappelle que c'est dans l'«Examen " du Cid que Corneille, en réfléchissant à la 
question de la «bienséance » au théâtre, et à ce qui autorise parfois de ne pas la respecter, écrit :

Si nous ne nous permettions quelque chose de plus ingénieux que le cours ordinaire de la passion, nos Poèmes ramperaient souvent, et les grandes douleurs ne mettraient dans le bouche de nos Acteurs que des exclamations et des hélas. ${ }^{7}$

13 Je me mets à la place de Racine, je lis cette phrase, j'y trouve un magnifique défi, quelque chose en somme comme un programme d'écriture. Corneille écrit : Nos Poèmes ramperaient si on ne mettait dans la bouche de nos acteurs que des exclamations et des hélas. Le défi consiste, se dit Racine, dans l'hypothèse que je risque sur l'intentio auctoris, à écrire un Poème dramatique où il n'y aurait que des exclamations et des hélas, et qui, toutefois, ne soit pas un poème rampant. Pour réussir une telle prouesse, il faut être habile. Racine relève le défi, il écrit Bérénice.

On trouve que je vais trop vite en besogne, le scénario que j'imagine, quand on veut bien me suivre dans mon raisonnement, n'a rien d'invraisemblable. J'ajoute qu'il existe une autre excellente raison permettant d'avancer que Racine a bien en tête la phrase de l' «Examen " au moment de préparer une pièce de théâtre sur un sujet qui sera également retenu, au même moment, par Corneille. C'est que Racine est l'ami de Boileau et qu'on doit à celui-ci une fameuse épigramme anti-cornélienne, qui a été publiée dans l'édition des CEuvres diverses de 1701 :

J'ai vu Agésilas

Hélas!

On sait que le même Boileau a ajouté dans un deuxième temps :

Mais après l'Attila

Holà. $^{8}$

Certes, ces textes n'ont été révélés au public qu'après la mort de Racine. Mais il est difficilement croyable que Boileau n'aurait pas parlé à son ami, dès 1666, donc dès la création d'Agésilas, de ces petits objets satiriques qu'il a fabriqués et qui, de toute manière, dans les salons, circulaient entre happy few. Je n'exclus pas que Racine ait pu jouer, à ce propos, un rôle d'instigateur. Georges Couton dans son édition des Euvres complètes commente le sens des textes: "Que Corneille ait pu écrire Agésilas est déplorable; mais après Attila, il faut qu'il prenne sa retraite ${ }^{9}$. Imaginons Racine parlant littérature avec Boileau, abordant, au coin du feu, les deux légèrement éméchés, le sujet des chers confrères. Le premier encourage le second, il lui donne une idée: Despréaux, on va se moquer de Corneille. Je sais un moyen. Et si tu faisais une épigramme? Ces conjectures, encore une fois, ne sont pas gratuites, elles s'appuient sur des faits. Si Racine a pu faire ce genre de suggestion à Boileau, qui, lui, fait rimer Agésilas avec Hélas, c'est qu'elle a ici encore sa source dans les textes. L'épigramme sur Agésilas, je le rappelle également, est une quasi-citation. Elle fait allusion à la réplique de Mandane, la sœur de Spritridate, dans l'acte IV :

Je n'entends pas des mieux

Comme il faut qu'un hélas s'explique

Et lorsqu'on se retranche au langage des yeux,

Je suis muette à la réplique. ${ }^{10}$

17 Imaginez que vous êtes Racine, que vous cherchez à ironiser sur Corneille, il y a là tout ce qu'il faut, vous n'avez qu'à ramasser ce que le hasard a réuni pour vous. L'auteur du Cid met en garde contre les "Hélas!", il pense, comme Voltaire, qu'ils sont une solution de facilité au théâtre et qu'il faut les éviter. Or voilà que le même Corneille est à son tour pris la main dans le sac, si l'on peut dire, cette fois par Boileau, dans une 
épigramme des Euvres diverses. Je m'identifie toujours à Racine, je dispose, avec ce dossier sur les " Hélas!», de suffisamment d'éléments pour écrire une pièce qui soit à la fois une tragédie esthétiquement à la hauteur, j'entendrai par là : qui parvienne à capter l'attention du public parce qu'elle est aussi hautement émouvante, et une œuvre polémique où, pour les bons entendeurs, il est expliqué pourquoi Racine est un plus grand auteur que Corneille.

18 4. Je fais intervenir, pour donner plus de force à mon argument, un troisième joueur. Il s'agit de l'abbé de Villars, auteur d'une Critique de Bérénice qui paraît à Paris au commencement de 1671 et qui annonce un peu déjà les observations que fera, au siècle suivant, Voltaire dans ses « Remarques sur Bérénice ». L'auteur de Candide a-t-il lu l'abbé de Villars? J'avoue que je n'en sais rien. Peu importe. Le fait est - je m'en tiendrai à cette affiliation pour l'instant - que l'on observe entre les textes une série de ressemblances. Pour Villars, comme pour Voltaire, Bérénice n'est pas une vraie tragédie. Racine, suggère l'auteur de la Critique, écrit pour ses fans, il fait pleurer les dames. Or on peut dire la même chose autrement. Racine, selon Villars toujours, n'est pas Corneille. Bérénice aurait été bien meilleure comme tragédie si Racine s'était conformé au modèle cornélien. Villars écrit donc :

L'Auteur a trouvé à propos pour s'éloigner du genre d'écrire de Corneille, de faire une pièce de Théâtre, qui depuis le commencement jusqu'à la fin, n'est qu'un tissu galant de Madrigaux et d'Elégies: et cela pour la commodité des Dames, de la jeunesse de la Cour, et des faiseurs de recueils de pièces galantes. ${ }^{11}$

19 Comme Voltaire toujours, Villars est frappé - comment ne pas l'être ? il y en a vingthuit au total ! - par la fréquence des « Hélas! ». Il en fait ici un sujet de moquerie :

Sans le Prince de Comagène qui est naturellement prolixe en lamentations et irrésolutions, et qui a toujours un toutefois et un Hélas de poche pour amuser le Théâtre, il est certain que toute cette affaire s'expédierait en un quart d'heure, et que jamais action n'a si peu duré. (Ibid.)

Il n'est question, dans ce que l'on vient de lire, que du prince de Comagène, donc d'Antiochus. Mais on admettra que Villars, comme Voltaire, vise la série, donc l'effet pléthorique. En incriminant les « Hélas! » d'Antiochus, Villars s'en prend à ce qui est à ses yeux un tic d'écriture, et aussi la maladresse, ou la paresse, ou l'insolence, ou tout cela à la fois d'un auteur qui serait donc, selon Villars, peu enclin à soigner son travail. On pourra dire avec les termes de l'« Examen » du Cid que, puisque les « Hélas!» sont omniprésents, pour Villars, le poème rampe.

L'expression « Hélas de poche » doit être clarifié. Je rappelle le commentaire de Georges Forestier :

Villars jouait sur les mots: un acteur qui représentait un personnage en pleurs sortait toujours un "mouchoir de poche » qu'il plaçait devant ses yeux, manière d'indiquer au public qu'il pleurait tout en affectant de masquer ses larmes [...] Les trois principaux personnages de la pièce devaient faire un abondant usage de ces "mouchoirs de poche », et l'on conçoit bien qu'Antiochus, en prononçant le dernier mot de la tragédie, qui est le dernier de ses quatre «Hélas!», avait en main son «mouchoir de poche ». Pour Villars, il était assurément difficile d'aller plus loin dans la dérision. ${ }^{12}$

21 L'attaque de Villars est précise, violente, habilement amenée. L'homme est un excellent satiriste $^{13}$. Mais tout cela n'est pas notre sujet, ni notre question. Notre question est: comment Racine réagit-il ? Or j'en viens à la réaction du dramaturge. Sans jamais désigner nommément l'auteur de la Critique, Racine répond à Villars dans une «Préface » placée en tête d'un volume qui paraît le 24 janvier 1671 et dont le titre est 
Bérénice, Tragédie, par M. Racine. J'apprends chez Georges Forestier toujours que c'est la première fois que Racine fait imprimer son nom sur une page de titre. On retiendra la volonté d'auto-affirmation. Je suis là. Je suis Racine. J'écris moi aussi des tragédies. En quelque sorte, la préface de 1671 est un manifeste et il s'agit d'un manifeste en faveur de la tragédie racinienne en ce qu'elle a de spécifique. Vous voulez savoir pourquoi Racine est plus fort que Corneille ? Lisez ou allez voir Bérénice! Villars croit pouvoir se moquer des « Hélas! ", Racine lui répond aussi sur ce point. L'abbé est alors renvoyé à son néant. Le pauvre Villars, nous explique-t-on, n'a rien compris à ce qu'est le théâtre, et il n'a pas compris ce qui fait, justement, l'originalité de Bérénice. Je cite Racine dans la «Préface » :

Et que répondrais-je à un Homme qui ne pense rien, et qui ne sait pas même construire ce qu'il pense [...] Il se plaint que la trop grande connaissance des Règles l'empêche de se divertir à la Comédie. Certainement si l'on en juge par sa Dissertation, il n'y eut jamais de plainte plus mal fondée. (Racine, op. cit. : 452)

Plus loin :

Mais je lui pardonne de ne pas savoir les Règles du Théâtre, puisque heureusement pour le Public, il ne s'applique pas à ce genre d'écrire. Ce que je ne lui pardonne pas, c'est de savoir si peu les Règles de la bonne plaisanterie, lui qui ne veut pas dire un mot sans plaisanter. Croit-il réjouir beaucoup les honnêtes Gens par ces Hélas de poche, ces Mesdemoiselles mes Règles, et quantité d'autres basses affectations qu'il trouvera condamnées dans tous les bons Auteurs, s'il se mêle jamais de les lire? (ibid.)

23 Cela peut s'appeler une exécution en bonne et due forme. Attrape l'abbé et disparais sous terre! Crève, vermine! Mais nous ne pouvons, dans les circonstances données, nous en arrêter là. Pour bien comprendre ce qui se passe, c'est-à-dire pourquoi Racine, par ce qu'il écrit, parvient à glorieusement clouer le bec à Villars, il faut citer aussi un passage de la «Préface » qu'on lit un peu plus haut, et qui a au demeurant fait couler beaucoup d'encre déjà. Villars a osé appeler Bérénice un « tissu galant de Madrigaux et d'Elégies ", Racine lui tire le tapis sous les pieds. Dans sa «Préface», il fait la défense d'une esthétique dramaturgique délibérément minimaliste. Au théâtre, écrit Racine, il faut être simple, et même le plus simple possible :

Il y a en a qui pensent que cette simplicité est une marque de peu d'invention. Ils ne songent pas qu'au contraire toute l'invention consiste à faire quelque chose de rien, et que tout ce grand nombre d'Incidents a toujours été le refuge des Poètes qui ne se sentaient pas dans leur Génie assez d'abondance, ni assez de force, pour attacher durant cinq Actes leurs Spectateurs, par une action simple, soutenue de la violence des passions, de la beauté des sentiments, et de l'élégance de l'expression. (ibid.)

Toute l'invention consiste à faire quelque chose de rien. La phrase est souvent rappelée. Pour ma part, je n'hésiterai pas à la mettre en rapport avec ce qu'on lit deux paragraphes plus loin, quand Racine s'occupe à clouer Villars, le malotru qui l'a attaqué sur ses « Hélas! », au pilori : que répondrais-je à un Homme qui ne pense rien ? Il faut, me semble-til, être attentif à la double allusion au rien, c'est-à-dire à l'effet d'écho que fait apparaître la «Préface ». Rien, chez Racine, ne veut évidemment pas dire rien chez Villars. Celui-ci, soutient Racine, ne pense rien, sa pensée et son discours sont parfaitement creux. D'ailleurs, l'homme n'écrit pas pour le théâtre. Encore heureux. S'il devait s'y mettre, ce serait la catastrophe! Villars est alors un repoussoir parfait pour ce que Racine veut ici nous apprendre sur lui-même. On peut, au théâtre, créer avec rien. Racine est un auteur de théâtre qui réussit dans ses pièces le miracle qui consiste à faire de rien quelque chose. Racine a du génie, Villars ne pense rien, n'est rien. 
5. Une autre question doit alors être posée, et on arrive ici au cœur du problème. Quel est donc ce rien que le dramaturge, par son art, qui n'est pas l'art de Corneille, qui doit sa valeur et sa spécificité à ce que, justement, Racine s'écarte de la norme cornélienne, parvient à transformer en quelque chose ? Il me semble que, avec les éléments dont nous disposons, la réponse à la question va à peu près de soi. Le rien de Bérénice est quelque chose. Le rien de Bérénice est un mot et ce mot est « Hélas! ». Villars croit faire de l'esprit en se moquant des «Hélas!», il passe à côté de l'essentiel, il est simplement bête. Voltaire se montre tout aussi aveugle. Pour qui sait y faire attention, en effet, les choses étaient parfaitement claires, dès le début ou presque. L'analyse qu'on a proposée plus haut de la scène 4 de l'acte II nous a donné notre point de départ, ensuite il a suffi de suivre les indications que donne le texte. Je rappelle la scène 7 de l'acte IV. Antiochus essaie de convaincre Titus. Bérénice, dit Antiochus, est malheureuse car elle ne comprend pas ce qu'on veut d'elle. Titus, selon Antiochus toujours, doit aller lui parler. Mais Titus, vide supra, ne peut parler, la seule chose qu'il puisse dire est qu'il ne peut rien dire. Cela n'invite guère à se montrer volubile. On entend d'abord, dans la scène en question, Antiochus qui prononce :

Allez, Seigneur, allez-vous montrer à sa vue.

Sauvez tant de vertus, de grâces, de beauté,

Ou renoncez, Seigneur, à toute humanité.

Dites un mot.

A quoi Titus répond, car Titus, qui ne peut rien dire, répond quelque chose :

Hélas! Quel mot puis-je lui dire? (IV, 7)

Titus, selon Barthes, est « aphasique ». Après la démonstration qui a été faite, je ne suis plus d'accord avec Barthes. En quelque sorte, tout est dit par ce que dit, ici, Titus. Lisons mieux le texte. Quel mot peut-il dire ? Le seul mot que Titus puisse dire, il le dit, et ce mot est «Hélas!». Le «Hélas!» dit tout. Intentio auctoris, avons-nous dit. L'intention d'auteur est clairement indiquée, pour la reconnaitre, on nous demande seulement de ne pas passer à côté de ce qu'on peut appeler d'une façon peut-être un peu pédante la sui-référentialité du vers 1236. Hélas! Quel mot puis-je lui dire? Comprenez par là : «Hélas!» est le seul mot que je puisse dire. Pour parler comme les logiciens, il y a là une confusion, que Racine introduit délibérément, entre usage et mention. Je dis : Hélas!, je cite «Hélas!». On ne sait pas si je m'exprime littéralement ou si je montre un objet langagier, que j'offre à la contemplation.

Villars et Voltaire, et avant eux Corneille, suggèrent que le « Hélas!» est une tare au théâtre, surtout quand on l'utilise à tort et à travers, chose que font les mauvais auteurs. Corneille, pour sa part, s'avance imprudemment et Boileau n'a aucune difficulté, sur la question des "Hélas!», à mettre l'auteur du Cid et d'Agésilas en contradiction avec lui-même. Arrive alors Racine. Son pari, dans Bérénice, est de faire mentir la règle que formule l'auteur de l' "Examen" du Cid en quelque sorte en la mettant dessus dessous. On peut faire une bonne pièce avec que des « Hélas! » et des exclamations. Toute l'invention consiste à faire quelque chose de rien.

6. Je vais vers une conclusion provisoire. Quand on y réfléchit, Racine, par la sorte de minimalisme radical qu'il revendique, ouvre aussi - peut-être le fait-il ici encore intentionnellement - un boulevard à de possibles réécritures "oulipiennes» de sa tragédie. Imaginons une Bérénice dont le seul texte serait le mot "Hélas!» inlassablement répété par les comédiens, et cela pendant cinq actes, comme le veulent les règles de la tragédie. Je suis assez enclin à penser que Racine a effectivement pu être 
tenté par ce type de dispositif, mais qu'il s'est finalement abstenu pour une raison que je crois aussi très bien comprendre. À son époque, les esprits ne sont pas encore mûrs pour un exercice de ce genre, Racine préfère laisser le champ libre aux générations futures ${ }^{14}$.

7. J'aurais voulu encore, mais je n'en ai pas le temps car les sévères rédactrices de Carnets m'attendent, ajouter quelques réflexions sur le statut grammatical du «Hélas!». Pour la critique dramaturgique, il y a là encore des observations intéressantes à faire. Le "Hélas!» appartient comme on sait à la catégorie des «interjections». Mon Petit Robert précise: "Interjection. Du lat. interjectio. Mot invariable pouvant être employé isolément pour traduire une attitude affective du sujet parlant». La catégorie des interjections, depuis toujours, fascine les grammairiens parce qu'on a l'impression qu'en quelque sorte elles sont «hors langage». Une interjection est un cri, plus proche de l'animal que de l'homme. On est, ou on croit être dans une logique de l'expression "primitive». Diderot, pour sa part, entrevoit une similarité entre les interjections, ou les exclamations, et les premières verbalisations : «De la liaison des passions avec des organes naissent les voix ou les cris ${ }^{15}$. Mais une telle conception " primitiviste » est de toute évidence sujette à caution, et c'est ce que grammairiens et linguistes ont également fait observer. L'effet de spontanéité que produit l'interjection est un effet du discours.

La linguiste Laurence Rosier, à propos du cas qui nous intéresse, écrit : "Dire hélas correspond à un recours à l'institué de la langue plutôt qu'à une information expressive. L'interjection fonctionne de façon codée, rituelle diraient les ethnologues $»^{16}$. Et l'on peut rappeler aussi les études consacrées au fonctionnement des interjections dans le cadre de la théorie " polyphonique » d'Oswald Ducrot : «L'être à qui est attribué le sentiment, dans une interjection, c'est $\mathrm{L}$, le locuteur vu dans son engagement énonciatif $»^{17}$.Christine Sirdar-Iskandar, élève de Ducrot, ajoute: "Par l'emploi de certaines interjections à valeur modalisatrice, l'énonciateur peut adopter des attitudes, jouer des rôles; ces interjections lui fournissent tout un assortiment de personnages : étonné (Tiens!), heureux (Chic! Tant mieux!), scandalisé (Oh!), soulagé (Ouf! Enfin! Ah!) »18. On voit très bien ici le lien avec le théâtre. Quand j'ai recours à une « interjection à valeur modalisatrice ", j'incarne un personnage, je joue un rôle, je suis en fait un comédien sur scène. Quand, par exemple, je dis « Hélas! », selon la théorie de Ducrot, j'incarne, devant mon interlocuteur, un personnage - en termes ducrotiens : un locuteur -, qui, lui, dans la sorte de théâtre discursif que mon interlocuteur et moi avons mis en place, se trouverait dans la situation où le «Hélas!" serait, de sa part, une intervention pertinente. Mais le locuteur doit être distingué de l'énonciateur, entre les deux, il y a la même distance qu'entre le comédien et son personnage. En somme donc, quand je dis «Hélas!", je suis comme Titus sur scène ou comme Bérénice, ou comme Antiochus. Le théâtre n'imite pas la vie, la vie imite le théâtre. Mais je le savais déjà, Racine m'a aussi appris cela. Je dis merci à Racine. Obrigado João. 


\section{NOTES}

1. Racine, Bérénice, in Cuvres complètes (1999). I, Théâtre-poésie, éd. présentée, établie et annotée par Georges Forestier, Paris : Gallimard « la Pléiade », p. 476.

2. Barthes, Sur Racine (1963). Paris : Seuil, p. 76.

3. Voir aussi les vers 569 (Bérénice), 600 (Titus), 610 (Bérénice), 616 (Titus).

4. Bryant C. Freeman, Alan Batson (1968). Concordance du Théâtre et des Poésies de Jean, Racine, tome I, Cornell University Press, p. 604 et suiv.

5. Euvres complètes, t. LV, p. 956, cité par Georges Forestier, Racine, Euvres complètes, op. cit., p. 1483.

6. Voir aussi la « Notice » de Georges Forestier, Racine, Euvres complètes, op. cit., p. 1446 et suiv.

7. Corneille, Euvres complètes (1980), textes établis, présentés et annotés par Georges Couton, « La Pléiade », t. I, p. 702.

8. Boileau, Euvres complètes, Paris: Gallimard, «La Pléiade", p. 248, cité par Georges Couton, p. 1518.

9. Corneille, Cuvres complètes. p. 1519.

10. Agésilas, IV, 4, CEuvres complètes, op. cit., p. 616.

11. Racine, Euvres complètes, op. cit., p. 516.

12. Jean Racine (2006), Paris Gallimard, «NRF Biographies », p. 409.

13. Mme de Sévigné écrit à propos de la plaquette de Villars : "Malgré cinq ou six mots qui ne valent rien du tout [...] cela est joli », lettre du16 septembre 1671, cité dans Jean Racine, p. 407.

14. Je renvoie aux exercices de «réduction de texte » que propose "l'Atelier» de Fabula, ou à la pièce contemporaine de Nicole Genovese, Hélas (2018), où, si je suis bien informé, les « Hélas! » que prononcent les personnages sont soulignés par des coups de cymbale.

15. Eléments de physiologie, 1774.

16. «Interjection, subjectivité, expressivité et discours rapporté à l'écrit : petits effets d'un petit discours ", Cahiers de praxématique, $\mathrm{n}^{\circ}$ 4, 2000, p. 3.

17. Oswald Ducrot (1984). Dire et ne pas dire, Paris : Minuit, p. 200.

18. «Eh bien! Le Russe lui a donné cent francs » dans Ducrot et al. (1980), Les Mots du discours, Paris, Minuit, p. 161.

\section{RÉSUMÉS}

Il s'agit d'une analyse des nombreux « Hélas !» dans Bérénice de Racine. On propose l'hypothèse selon laquelle ce grand nombre a été voulu par l'auteur qui cherche ainsi à dégager ce qui est, à ses yeux, la quintessence du genre tragique.

This is an analysis of the frequent use of the interjection « Hélas! » in Bérénice by Racine. The idea is that the effect is intentional (intentio auctoris). Racine has deliberately multiplied the « Hélas! » to obtain a certain effect, to explore the essence of the tragic discourse. 
INDEX

Keywords : Racine (Jean), Bérénice, Hélas !, Intentio auctoris, tragic, tragedy

Mots-clés : Racine (Jean), Bérénice, Hélas !, Intentio auctoris, tragédie, tragique

\section{AUTEUR}

FRANC SCHUEREWEGEN

Université d'Anvers

franc.schuerewegen[at]uantwerpen.be 